\title{
Longitudinal beam splitting and coalescing using an off-momentum drifting barrier bucket
}

\author{
T. Yoshimoto $\odot,{ }^{1}$ M. Hirose,${ }^{1,2}$ X. Liu, ${ }^{3}$ K. Takayama, ${ }^{1}$ T. Adachi,,${ }^{1}$ K. Okamura, ${ }^{1}$ E. Kadokura, ${ }^{1}$ \\ T. Kawakubo, ${ }^{1}$ S. Takano, ${ }^{1}$ and N. Munemoto ${ }^{1,4}$ \\ ${ }^{1}$ High Energy Accelerator Research Organization (KEK), 1-1 Oho, Tsukuba, Ibaraki 305-0801, Japan \\ ${ }^{2}$ Tokyo City University, 8-9-18, Setagaya, Tokyo 158-8557, Japan \\ ${ }^{3}$ China Spallation Neutron Source (CSNS), Institute of High Energy Physics, 1 Zhongziyuan Road, \\ Dalang, Dongguan, Guangdong 523000, China \\ ${ }^{4}$ Tokyo Institute of Technology, 4259 Nagatsuta, Kanagawa 226-8503, Japan
}

(Received 28 May 2020; revised 5 March 2021; accepted 31 August 2021; published 11 October 2021)

\begin{abstract}
There has been much research on longitudinal bunch splitting and coalescing for accelerator performance improvements in rf synchrotrons. In this paper, we report a scheme with several induction cells that goes beyond the limitation in which the phase drift speed of beam buckets must be much lower than that of the maximum off-momentum particles to minimize longitudinal emittance blowup. In principle, additional pulse acceleration voltages can be applied to produce a momentum jump of the beam and save beam manipulation time, which is crucial for fast-cycling synchrotrons with limited injection and extraction times. This paper compares the new and conventional schemes with experimental results. Finally, the beam behaviors are discussed with macroparticle simulations.
\end{abstract}

DOI: 10.1103/PhysRevAccelBeams.24.100401

\section{INTRODUCTION}

Longitudinal beam manipulation schemes in synchrotrons have been traditionally called rf gymnastics. Several schemes have been proposed and demonstrated, mainly at high-energy accelerator laboratories, to satisfy various demands on beam pulse shape. Radio frequency gymnastics is usually realized by a combination of adiabatic and nonadiabatic rf handling with continuous sinusoidal acceleration waveforms at different frequencies. Alternatively, rf gymnastics can also be achieved by a method called barrierbucket handling, which uses isolated acceleration pulses with wideband $\mathrm{rf}$ cavities. In 1983, Griffin et al. first reported barrier-bucket handling to achieve beam gap preservation for beam transfer [1]. Since then, barrierbucket handling has been widely researched. For instance, Bhat $e t$ al. at the Fermilab have proposed and demonstrated several attractive barrier-bucket schemes with rectangular and triangular acceleration pulses by wideband rf cavities. A well-summarized overview of these wideband beam handling schemes is given in Ref. [2]. Fast bunch cogging, in which longitudinal beam relocation is achieved through an off-momentum drifting barrier-bucket scheme (offDBB), is described in Sec. IV-d-iii of Ref. [2], and is

Published by the American Physical Society under the terms of the Creative Commons Attribution 4.0 International license. Further distribution of this work must maintain attribution to the author(s) and the published article's title, journal citation, and DOI. similar to our proposed scheme in that both employ an offmomentum beam bucket. However, our scheme realizes longitudinal beam splitting and coalescing through offmomentum beam handling. Several barrier-bucket studies were conducted at the Alternating Gradient Synchrotron $[3,4]$. The above barrier-bucket handling has been mostly realized with $\mathrm{rf}$ cavities energized by solid-state power amplifiers.

Another approach to barrier-bucket handling and acceleration is to use induction cells energized by switching power supplies, in which pulse timings are controlled by a digital signal processor or a field-programmable gate array (FPGA). At the High Energy Accelerator Research Organization (KEK), beam acceleration and manipulation schemes have been extensively developed since the first demonstration of the induction synchrotron at the $12-\mathrm{GeV}$ proton synchrotron with induction cells [5-9], and the KEK digital accelerator (KEK-DA) [10-14], which is a fastcycling induction synchrotron.

To improve beam operation capabilities of the KEK-DA to satisfy various beam demands, we propose a novel scheme for fast longitudinal beam splitting and coalescing. The scheme can control the number of charged particles and the length of a bunch at any acceleration stage (injection, middle of acceleration, and extraction) through off-momentum bucket handling. For example, we can send several split beams to different locations in one acceleration cycle, rendering this capability cost efficient for industrial use. Another application of our proposed scheme is for superbunch acceleration [13]. The injected beam of the 
KEK-DA is a continuous $50 \mathrm{keV} /$ nucleon ion beam. Although our induction cells have a low quality factor of $0.1-0.2$, there exists a mechanical limitation due to droops in the long acceleration pulses [7]. The maximum length of a flat acceleration pulse is $\sim 4 \mu \mathrm{s}$, whereas the typical beam revolution period of the KEK-DA at injection is $>10 \mu \mathrm{s}$. Therefore, we must split a long injected beam into several superbunches while minimizing the beam loss and manipulation time.

Typical longitudinal beam splitting and coalescing schemes intrinsically include beam relocation with on-momentum moving buckets. We refer to these wellestablished schemes as on-momentum drifting barrierbucket schemes (onDBB).

It is crucial to minimize longitudinal emittance blowup through these approaches. Adiabatic beam handling, where the speed of the drifting beam bucket is much lower than the phase drift speed of the maximum off-momentum particles in the longitudinal phase space, effectively avoids emittance blowup. However, beam handling using barrier buckets is not adiabatic in principle because particles near the synchronous line or on-momentum line never move. Any relocation process acts nonadiabatically on these particles. $\mathrm{Ng}$ [15] has given the criterion for $\mathrm{rf}$ bucket handling to achieve adiabaticity in the longitudinal motion as

$$
\text { Barrier drifting time } \gg \frac{2 T_{\mathrm{r}}}{|\eta \delta|},
$$

where $T_{r}$ is the beam relocation length, $\eta$ is the momentum slippage factor, and $\delta$ is the relative momentum spread of the beam. Strictly speaking, the criterion should be regarded as the adiabaticity criterion for a particle with momentum deviation $\delta$. Some emittance degradation caused by this intrinsic nonadiabatic feature is inevitable in onDBB. At the injection energy of the KEK-DA operated at $10 \mathrm{~Hz}, \eta$ is $-0.81, \delta_{\max }$ is $3.4 \times 10^{-3}$, and $T_{r}$ is approximately $10 \mu \mathrm{s}$, resulting in $2 T_{r} /|\eta \delta| \approx 7.3 \mathrm{~ms}$. Therefore, it is too difficult to satisfy criterion (1) because of the acceleration period of $50 \mathrm{~ms}$, and the formation of several superbunches in the KEK-DA appears to be infeasible. A quicker beam manipulation scheme is essential to achieve longitudinal beam splitting and relocation with minimum beam loss and emittance blowup. Beam loss minimization is crucial even at low energies $(\sim 50 \mathrm{keV} /$ nucleon $)$ to prevent metal ion beams from being deposited on the surface of electrical insulators such as ceramics.

To meet these demands, a bucket is instantaneously shifted to an off-momentum position in the longitudinal phase space. The off-momentum kick plays an important role in this offDBB and is the difference between the offDBB and onDBB. In this paper, we propose and experimentally demonstrate this scheme as a proof-ofprinciple experiment. In the experiment, an injected beam was split into two bunches at the beginning, and then one of the two split bunches was longitudinally manipulated, while the other was steadily captured in a bucket. Subsequently, the two divided beams coalesced into one beam through a sophisticated acceleration pulse manipulation. All the acceleration pulse timings for the beam handling were preprogrammed and controlled by an FPGA.

This paper is organized as follows. The concept of the offDBB is presented in Sec. II, focusing on the differences compared with the onDBB. Experimental setup and results are presented in Sec. III. In Sec. IV, the experimental results are analyzed and discussed with the help of macroparticle simulations in detail. The results are summarized with future perspectives in the final section.

\section{COALESCING AND SPLITTING SCHEMES}

This section explains the longitudinal beam coalescing processes in the onDBB and offDBB. Beam splitting manipulation is viable in each time-reversal process.

\section{A. On-momentum drifting barrier-bucket scheme}

The conventional onDBB is illustrated in Fig. 1. First, the two buckets separately confine bunches No. 1 and No. 2 [Fig. 1(a)]. Next, the barrier voltages for bunch No. 2 are gradually triggered at an earlier timing, and the right bucket moves towards the left. Bunch No. 2 is eventually close to bunch No. 1 [Fig. 1(b)] with some emittance blowup, which depends on the drift speed of the bucket. At the last stage, bunches No. 1 and No. 2 coalesce into one bunch after the barrier voltages are turned off [Figs. 1(c) and 1(d)]. This final process causes additional emittance blowup due to the finite gap between the two bunches [Fig. 1(c)]. Bunch splitting manipulation is also viable in the inverse process.

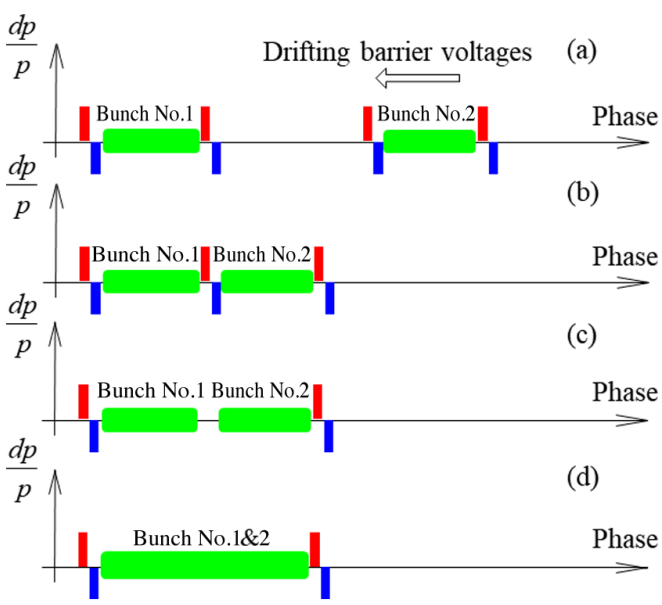

FIG. 1. Beam manipulation in the onDBB. Positive and negative voltages are in red and blue, respectively. (a) Bucket voltages confine bunches No. 1 and No. 2, and the right bucket moves towards the left. (b) Bunch No. 2 is adjacent to bunch No. 1. (c) The intermediate voltages are turned off to combine bunch No. 1 with bunch No. 2. (d) The long bunch is confinable with the new barrier voltages. 
In both cases, the emittance blowup is strongly affected by the drift speed. In Secs. III and IV, we discuss beam motion in the onDBB with the experimental results and simulations.

\section{B. Off-momentum drifting barrier-bucket scheme}

An offDBB is illustrated in Fig. 2. The offDBB is similar to the onDBB, with the exception that momentum jumps are introduced. First, the two bunches are separately captured in buckets. Next, bunch No. 2 is given energy by a long acceleration pulse [Fig. 2(b)], jumps in the momentum space, and then spontaneously drifts due to the momentum difference. The longitudinal diffusion of drifting bunch No. 2 is suppressed with the drifting barrier voltages [Fig. 2(c)]. The trigger timings of the barrier voltages are uniquely determined by the drift speed of bunch No. 2. The amplitude of the long acceleration pulse must be appropriately chosen from the momentum slippage factor of the machine. Before overlapping bunch No. 1,

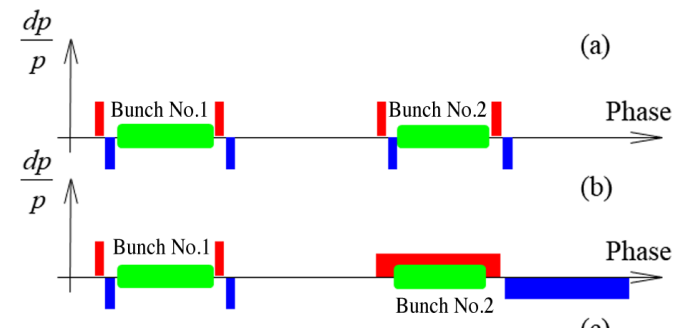

(c)

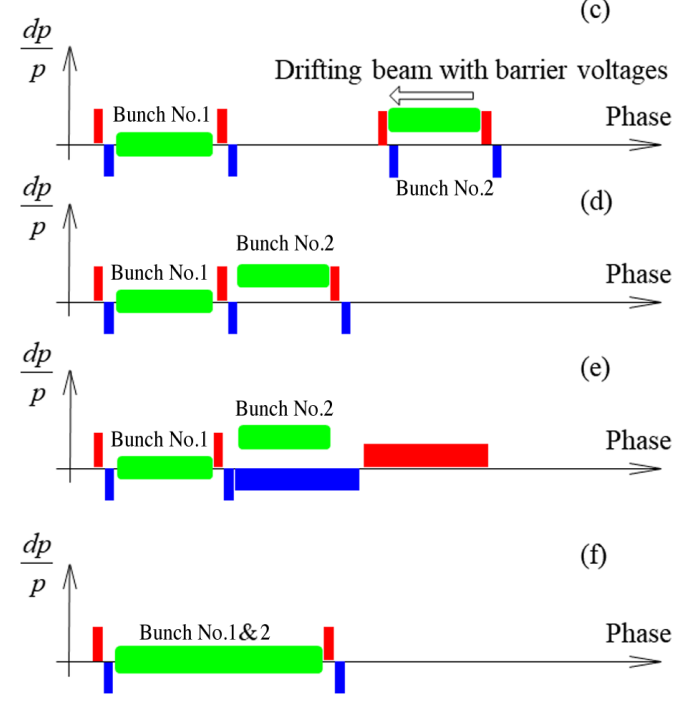

FIG. 2. Beam manipulation in the offDBB. Positive and negative voltages are in red and blue, respectively. (a) Bucket voltages confine bunches No. 1 and No. 2. (b) Bunch No. 2 is accelerated by a long positive voltage pulse. (c) Bucket voltages confine bunches No. 1 and No. 2, and the right bucket moves towards the left. (d) Bunch No. 2 is adjacent to bunch No. 1. (e) Bunch No. 2 is decelerated by a long negative voltage pulse and becomes on-momentum. (f) Bunches No. 1 and No. 2 are combined after the intermediate voltages are turned off, and the new long bunch is confinable with the new barrier voltages. bunch No. 2 loses energy by a long deceleration pulse, the amplitude of which is the same as that of the acceleration pulse at the stage in Fig. 2(b). After the deceleration, bunches No. 1 and No. 2 are on-momentum in the phase space and they gradually coalesce into a long bunch. The longitudinal emittance blowup is expected to remain low compared with that of the onDBB, although the low emittance growth is caused by the clearance process of the barrier pulses [Fig. 2(e)].

It is essential in the offDBB that the additional acceleration proactively assists the fast drifting of a bunch along the time axis. This enables adiabatic phase drifting. The dimensionless drift velocity $v$ on the phase axis is defined by $v=\eta \Delta p / p$ or $\eta \Delta E /\left(\beta^{2} E\right)$, where $\Delta E$ is the energy gain from the acceleration, $E$ is the total energy of an onmomentum particle, $\Delta p / p$ is the momentum deviation, and $\beta$ is the relativistic velocity. When the acceleration voltage $V_{0}$ is applied at several turns, $v$ discretely increases because $\Delta E=n Q e V_{0}$, where $n$ is the turn number for the acceleration with charge state $Q$, and $e$ is the unit charge. The longitudinal phase shift of bunch No. 2 is usually negligibly small during the necessary time $n T_{0}\left(T_{0}\right.$ is the revolution period) for this energy jump because $n T_{0}$ is much less than the time of the beam drifting just after the energy jump. The phase shift per turn of the beam, $\Delta \phi$, after the desired energy gain is achieved, is described by $2 \pi v$; thus, the time shift (corresponding to the longitudinal beam phase shift) per turn is expressed as $v T_{0}$ [s/turn].

\section{EXPERIMENTAL RESULTS}

\section{A. Setup}

A $75-\mu \mathrm{A} \mathrm{He} \mathrm{He}^{1+}$ beam was generated in the electroncyclotron-resonance ion source and directly injected into the ring. The length of a beam chopped by the Einzel lens chopper [16] was $4 \mu \mathrm{s}$. The beam revolution period was constant at $12.1 \mu \mathrm{s}$. With a beam of a few hundred $\mathrm{keV}$, a vacuum pressure of $\sim 10^{-6} \mathrm{~Pa}$ inevitably causes considerable beam loss due to the large cross section for electron capture, as discussed in Ref. [14]. We applied the static barrier-bucket scheme (SBB), onDBB, and offDBB to a $4-\mu \mathrm{s}$ beam and compared the results.

\section{B. Static barrier-bucket trapping}

The SBB is a type of beam confinement scheme in which two barrier pulses are placed at the edges of a beam to prevent the beam's longitudinal diffusion caused by a finite momentum spread and the longitudinal space charge effect. The measured data for the SBB, onDBB, and offDBB are shown in Fig. 3. The evolution of the beam intensity and voltage profiles are described with a time-turn plot with the inner time $t_{\text {in }}$ for one revolution period on the horizontal axis and the turn number after the injection on the vertical axis, as shown in Figs. 3(a)-3(c) and 3(d)-3(f), respectively. We set up the configuration of the beam diagnostic 


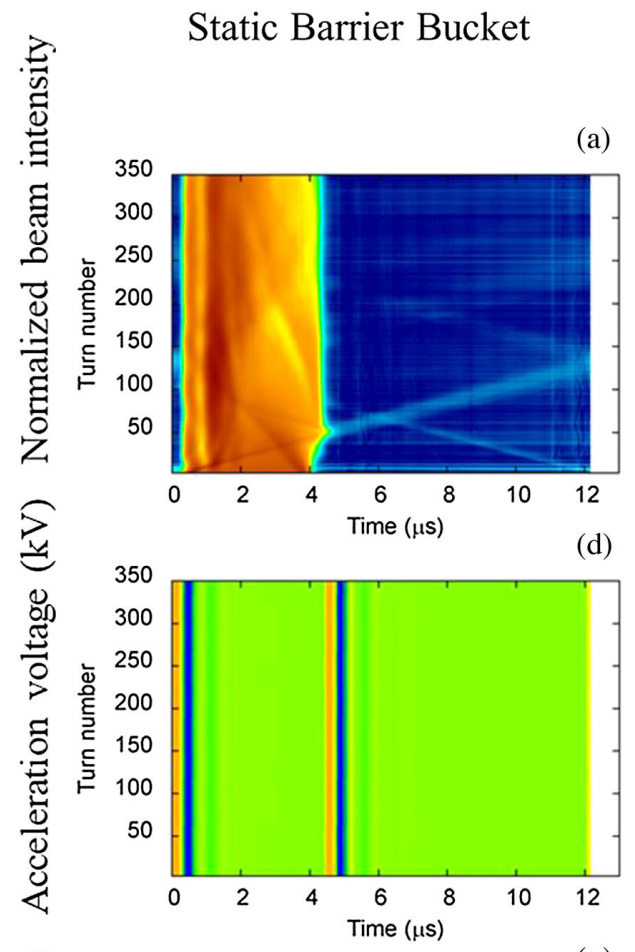

(g)

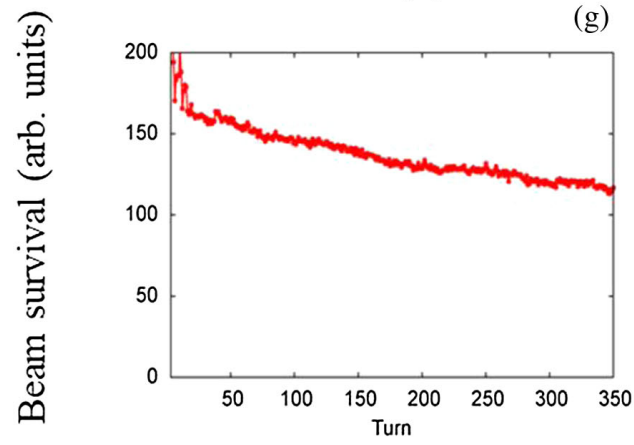

\section{On-momentum Drifting Barrier Bucket}

a)

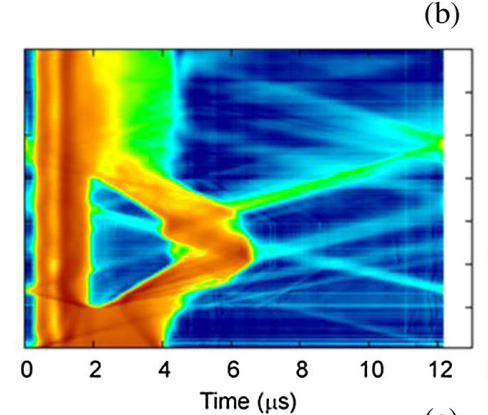

(e)

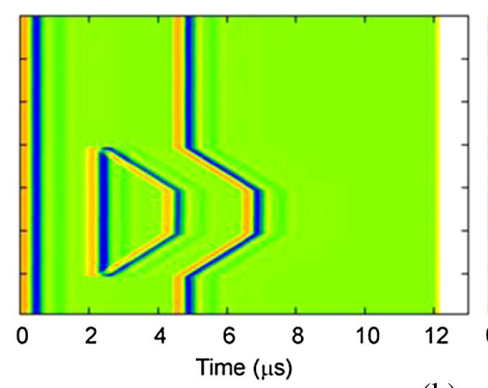

(h)

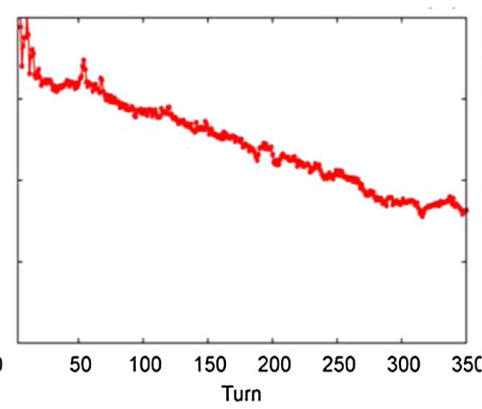

b)

\section{Off-momentum Drifting} Barrier Bucket

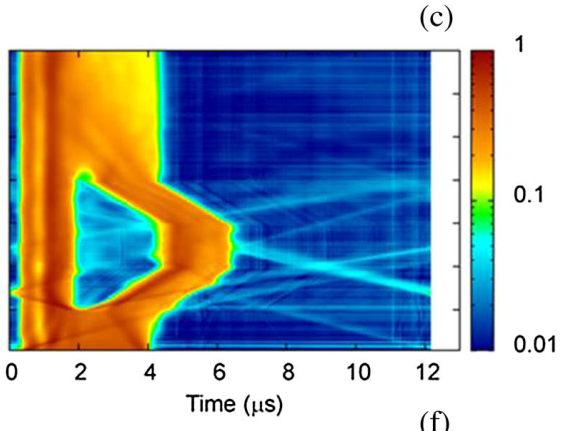

(f)

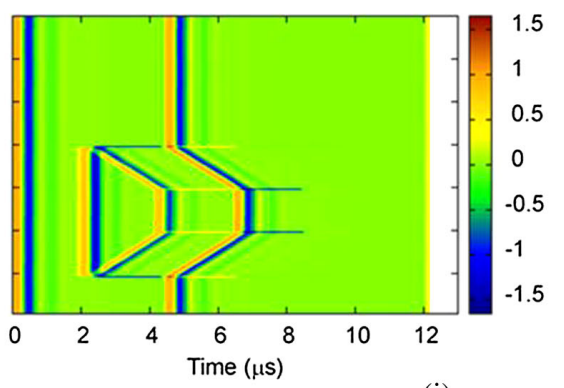

(i)

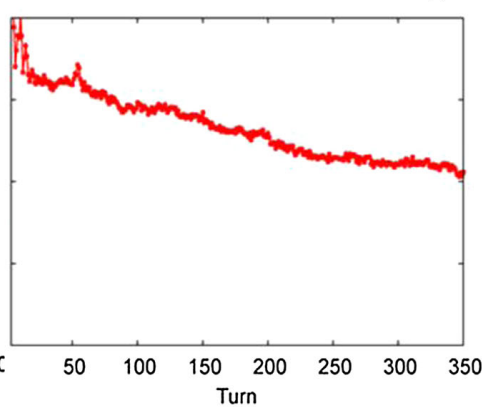

FIG. 3. Comparison of (top) beam intensities, (middle) acceleration voltages, and (bottom) beam survival for the SBB (left column), onDBB (middle column), and offDBB (right column) in the experiments. In the color-coded plots (a)-(f), the horizontal and vertical axes indicate the inner time in one revolution period and the turn number after the injection, respectively. In the plots (g)-(i), the horizontal and vertical axes denote the turn number after the injection and the beam survival in arbitrary units, respectively.

system for precise beam profile measurement with a $30-\mathrm{kHz}$ high-pass filter to suppress electric ground noise. However, the acceleration voltage profiles were unfavorably filtered due to the common diagnostic system. Therefore, we reconstructed higher precision acceleration waveforms from the recorded pulse timings and separately measured acceleration waveforms, which were unfiltered and used for beam simulations in Sec. IV [Figs. 3(d)-3(f)]. The beam survivals calculated from Figs. 3(a)-3(c) are plotted as a function of turn number in Figs. 3(g)-3(i).

In the SBB, two induction cells were employed to form a $4-\mu$ s beam bucket. Each induction cell produced a bipolar pulse (amplitude $V_{\mathrm{amp}}=1.0 \mathrm{kV}$, pulse width $t_{w}=250 \mathrm{~ns}$, and interval width $t_{\mathrm{int}}=150 \mathrm{~ns}$ ) [Fig. $13 \mathrm{of}$ the Appendix A]. Table I in Appendix A summarizes the parameters for the SBB. The faint fluctuating lines at $t_{\text {in }}=$ $\sim 1.0$ and $\sim 5.5 \mu \mathrm{s}$ in Fig. 3(d) are small voltage waveform distortions caused by the impedance mismatch of the induction acceleration system. These distortions affected the actual longitudinal beam behavior [Fig. 3(a)]. Figure 3(g) shows the evolution of the beam survival in the SBB. In the 0th-10th turns, $16 \%$ beam loss was observed; previous studies [16] revealed that the steep momentum distortion at the beam edges generated by the Einzel lens chopper causes this beam loss effect. The final beam survival ratio, excluding the initial beam loss in the first ten turns, was 0.69.

\section{On-momentum drifting barrier-bucket scheme}

First, the barrier pulses $\left(V_{\mathrm{amp}}=1.0 \mathrm{kV}\right.$ for induction cells No. 1 and No. 2) trapped a $4-\mu$ s beam in the first 47 turns, similar to the SBB [Fig. 3(e)]. At the 48th turn, we introduced two intermediate voltages $\left(V_{\mathrm{amp}}=1 \mathrm{kV}\right.$ for induction cell No. 3 and $V_{\mathrm{amp}}=0.81 \mathrm{kV}$ for induction cell 
No. 4), and two 2- $\mu$ s buckets were formed to split the beam into two bunches and capture them. The voltage $V_{\mathrm{amp}}=$ $0.81 \mathrm{kV}$ for induction cell No. 4 , which was $<1 \mathrm{kV}$, was attributed to the technical limitations of the current induction acceleration system, and the assisting voltages for the offDBB were also $0.81 \mathrm{kV}$. Through the trigger timing control of the acceleration pulses, the right beam bucket moved to the right until the 97th turn. The right bucket remained steadily positioned through the 98th-147th turns and gradually moved closer to the left bucket through the 148th-197th turns. Table II in Appendix A summarizes the trigger timings and amplitudes of the acceleration pulses for the onDBB. In the onDBB for beam splitting and coalescing, we observed considerable beam fluctuation and spill.

The final beam survival ratio, excluding the initial beam loss in the first ten turns, was 0.48 , which is $30 \%$ lower than that of the SBB [Fig. 3(h)]. This low survival ratio can be explained by the violation of Ng's criterion in Eq. (1). Note that we calculated the beam survival ratio from the integrated beam quantity within the beam buckets. The beam spill from the bucket region during the round trip of the bucket is visible in Fig. 3(b).

\section{Off-momentum drifting barrier-bucket scheme}

The offDBB applies quick momentum jumps to a barrier-trapped bunch. To achieve the momentum jumps, $2-\mu \mathrm{s}$ pulses were introduced; the amplitude $V_{\mathrm{amp}}$ was $-0.81 \mathrm{kV}$ (deceleration) in the 46th-47th and 198th199th turns, $0.81 \mathrm{kV}$ (acceleration) in the 98th-99th and 148th-149th turns. Due to FPGA programming issues, we applied the assisting voltages in the 46th-47th turns, instead of the 48th-49th turns, which was the original configuration; however, this two-turn shift was negligible. The reason for the two-turn momentum jump instead of a one-turn momentum jump is due to the technical constraint of the finite acceleration voltage height of $<1.2 \mathrm{kV}$. In Fig. 3(c), the appropriate off-momentum jump supported the natural drifts of the right bunch in the 48th-97th and 148th-197th turns. From the assisting voltage amplitude $V_{\text {amp }}=v \beta^{2} E /(|\eta| n Q e)=0.81 \mathrm{kV}$, the desired dimensionless drift velocity $v$ of the bucket was calculated to be $3.29 \times 10^{-3}[1 /$ turn $]$. The trigger timings and amplitudes of acceleration pulses for the offDBB are summarized in Table III in Appendix A.

The final beam survival ratio, excluding the initial beam loss in the first ten turns, was 0.62 , which is $10 \%$ lower than that of the SBB [Fig. 3(i)] and 29\% higher than that of the onDBB. The offDBB substantially reduced beam loss compared with the onDBB, as seen in Figs. 3(h) and 3(i), although it was still lower than that of the SBB. Further discussion is provided in Sec. IV. D.

\section{ANALYSIS AND DISCUSSION}

To examine the longitudinal beam behavior in each scheme, particle tracking simulations were performed with $10^{5}$ macroparticles. All the measured acceleration voltage profiles were used to evaluate the effects of the actual distortions, such as reflections by impedance mismatch. The longitudinal space charge effect was also implemented as a longitudinal beam impedance. The longitudinal beam dynamics is expressed by a set of discrete equations for energy $E$ and phase $\tau$ such that

$$
\begin{gathered}
E_{n+1}=E_{n}+Q e\left[V_{\mathrm{bb}}\left(\tau_{n}\right)+V_{\mathrm{acc}}\left(\tau_{n}\right)-\frac{Z_{0} g R}{2(\beta \gamma)^{2} c} \frac{\partial I_{\mathrm{b}}}{\partial t}\right], \\
\tau_{n+1}=\tau_{n}+2 \pi \cdot \eta_{n+1} \frac{E_{n+1}-\left(E_{\mathrm{s}}\right)_{n+1}}{\left(\beta_{n+1}\right)^{2}\left(E_{\mathrm{s}}\right)_{n+1}},
\end{gathered}
$$

where $V_{\mathrm{bb}}$ is the confinement voltage, $V_{\mathrm{acc}}$ is the acceleration voltage, $Z_{0}$ is the free space impedance $(377 \Omega), g$ is the geometric factor (6.417), and $R$ is the average radius of the ring $(6.0 \mathrm{~m}) . \gamma$ is the Lorentz factor, $c$ is the light speed, $\partial I_{b} / \partial t$ is the time derivative of the beam current term, and $E_{s}$ is the reference beam energy. The subscript $n$ indicates the turn number. With 360 bins, we calculated the longitudinal space charge effects with a central sixth-order differential and linear interpolation. The threshold lines for beam loss were set at the momentum deviation $\Delta p / p=$ \pm 0.012 to reproduce the experimental results. The macroparticle beam initially had a $4-\mu$ s uniform distribution and a momentum spread of $0.175 \%$ as $1 \sigma$ of the Gaussian distribution.

\section{A. Barrier-bucket trapping}

The evolution of the simulated beam intensity profile and phase-space beam distribution in the SBB are shown in Figs. 4 and 5, respectively. The simulation results reproduced the good confinement of the beam in the bucket. At $t_{\text {in }}=\sim 0.5 \mu \mathrm{s}$, the microstructure of the beam bucket trapped part of the beam, which was experimentally observed [Fig. 3(a)]. There was a small longitudinal

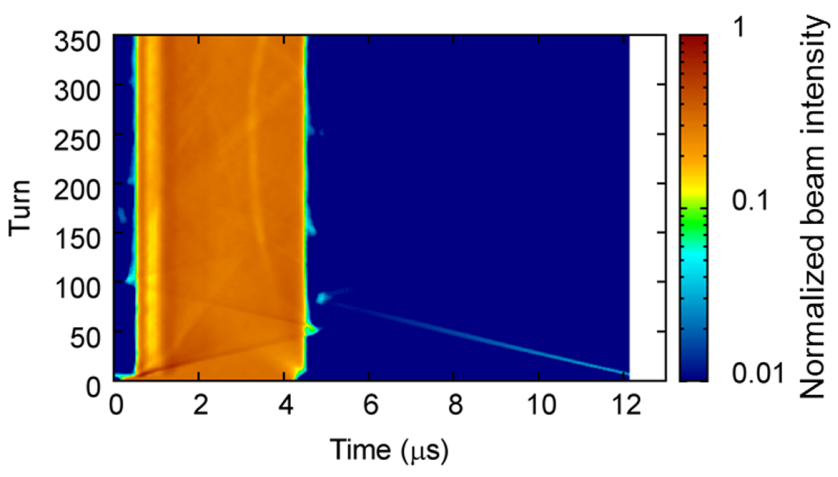

FIG. 4. Evolution of the beam intensity profile in the SBB (simulation). 

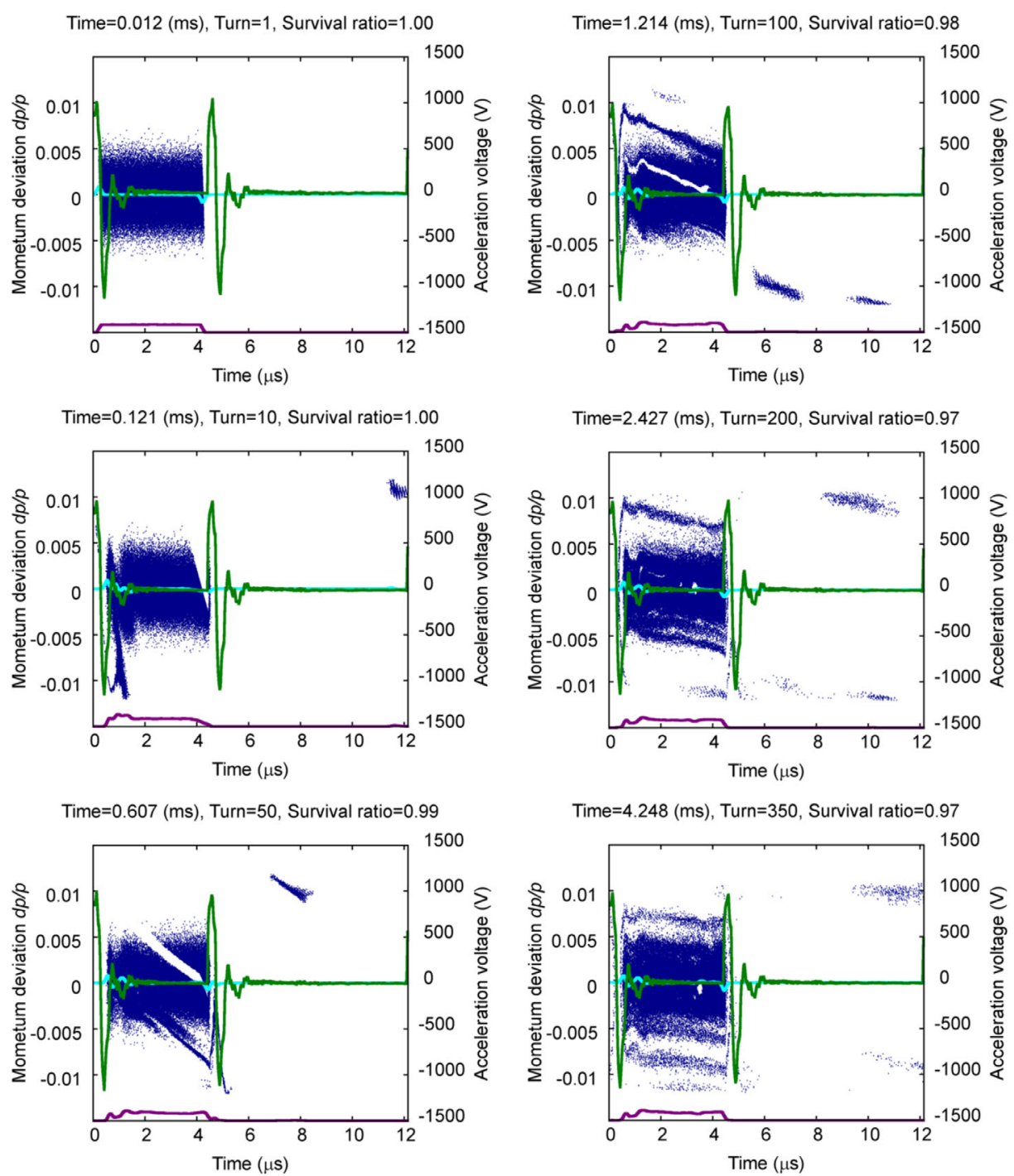

FIG. 5. Snapshots of the beam distribution in the longitudinal phase space in the SBB at the 1st, 10th, 50th, 100th, 200th, and 350th turns, from the top left to the bottom right. The barrier voltage and space-charge-induced voltage are indicated in green and cyan, respectively. The beam intensity profile in arbitrary units is indicated in purple.

mismatch between the injected beam and the waiting bucket location where the barrier pulses overlapped the bunch head. Hence, a small amount of the accelerated beam moved left and then appeared from the right, while the decelerated beam moved backward and was kicked forward by the second barrier voltages at the 50th turn. Figure 5 shows the overlapping effects in detail. The 10th and 50th turn snapshots in Fig. 5 explain the beam spills at $t_{\text {in }}=$ $\sim 0.5$ and $\sim 5 \mu \mathrm{s}$ in Fig. 3(a), respectively. At the 350th turn, a beam spill out of the $4-\mu$ s bucket was observed, and it was the primary source of beam loss in the SBB. The final beam survival ratio, excluding the effects of residual gas interactions, was 0.97 in this simulation.

Another interesting beam behavior was the momentum diffusion in the bucket caused by a series of small, sharp microstructures, and the steepness of the primary bucket waveforms. The combination of a high slippage factor, discrete acceleration, and complicated microstructures resulted in this momentum diffusion. Charged particles cannot continuously see acceleration fields when traveling along an entire ring circumference because of the finite length of the acceleration section. Thus, beam synchrotron motion inevitably causes this effect, depending on the slippage factor. To confirm this effect, we simulated the zero space-charge case with a fixed voltage profile over 350 turns for simplicity. The Poincare plots for the two test particles are depicted in Fig. 6. The injection and final positions of the two particle trajectories are indicated by squares and circles, respectively.

We can obtain the equipotential contours of the Hamiltonian as

$H\left(t_{\mathrm{in}}, \frac{\Delta p}{p}\right)=\frac{\eta \cdot E \cdot \beta^{2}}{2}\left(\frac{\Delta p}{p}\right)^{2}-\frac{Q e}{T_{0}} \int_{0}^{t_{\mathrm{in}}} V(\tau) d \tau$, 


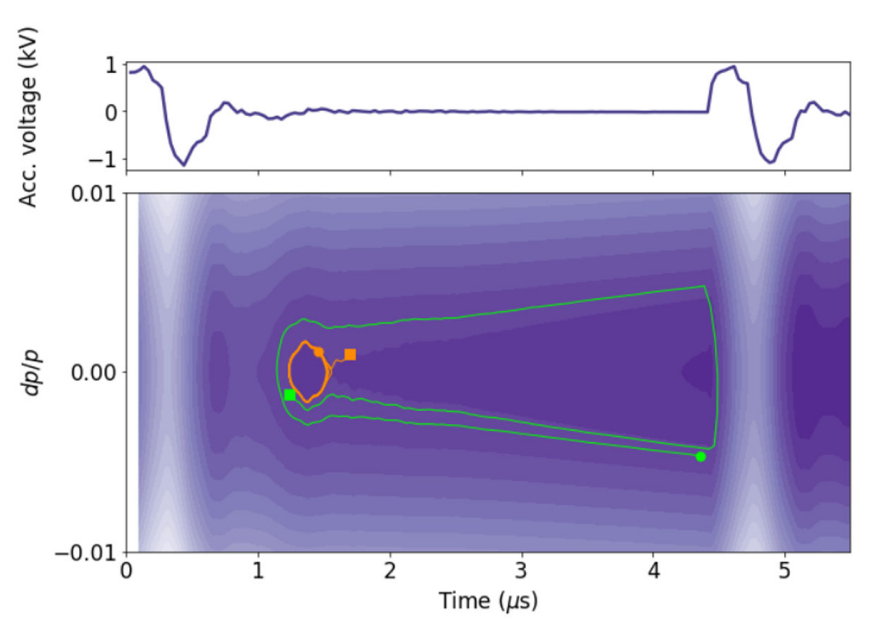

FIG. 6. Poincare plot of the two particle trajectories inside a fixed bucket over 350 turns. The orange and green particles move to the inner and outer equipotential lines, respectively.

where $t_{\text {in }}$ is the time corresponding to the longitudinal phase $\tau$ and $V$ is the acceleration voltage. During the 350 turns, one particle on the time-momentum plane $\left(t_{\text {in }}\right.$, $\Delta p / p)$ moved along the orange line from $(1.70 \mu \mathrm{s}$, $\left.1.02 \times 10^{-3}\right)$ to $\left(1.45 \mu \mathrm{s}, 1.17 \times 10^{-3}\right)$ on a lower equipotential line. In contrast, the other particle moved along the green line from $\left(1.24 \mu \mathrm{s},-1.23 \times 10^{-3}\right)$ to $(4.36 \mu \mathrm{s}$, $-4.66 \times 10^{-3}$ ) on a higher equipotential line. Hence, some particles had less momentum deviation, whereas the others had more momentum deviation via sophisticated discrete kicks through the entire bucket trapping, and the average momentum deviation statistically increased turn by turn. The same mechanism occurred in the onDBB and offDBB.

\section{B. On-momentum drifting bucket scheme}

The evolution of the beam intensity profile and phasespace beam distribution in the onDBB are plotted in Figs. 7 and 8 , respectively. The beam behavior was the same as that in the SBB until the 47th turn. After the 47th turn, the 2- $\mu \mathrm{s}$ bunch No. 2 was split out from the original $4-\mu$ s bunch and

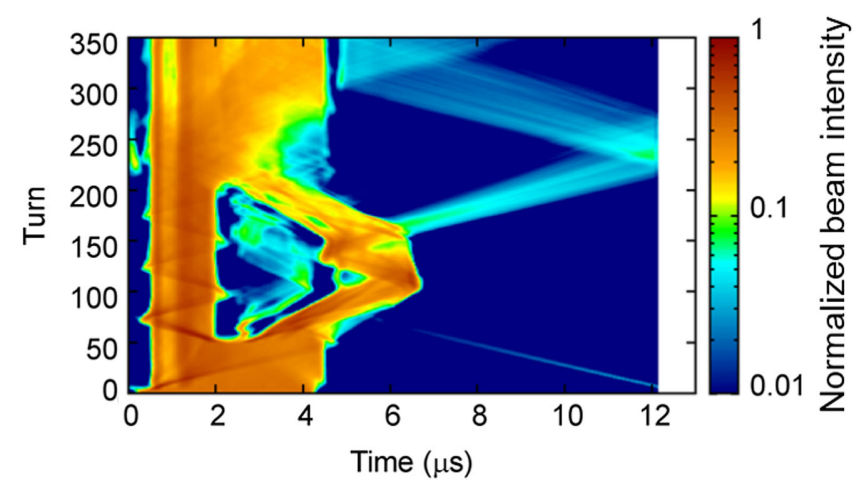

FIG. 7. Evolution of the beam intensity profile in the onDBB (simulation). guided towards the right by the emergence of the drifting bucket around $t_{\text {in }}=2 \mu$ s [Fig. 3(e)].

We observed three interesting beam phenomena in this simulation. First, bunch No. 1 was well confined in the 150 turns after the emergence of the intermediate voltages. However, the voltages imparted a sizable longitudinal oscillation to part of bunch No. 1 (see the 67th turn snapshot in Fig. 8). Next, a tiny amount of the beam was trapped between the two intermediate voltages (shown as the red line) in the 48th-197th turns in Fig. 8. This phenomenon was barely visible at $\left(t_{\text {in }}, n\right)=(2 \mu \mathrm{s}, 50)$ in Fig. 3(b). This trapping was inevitable because these particles were captured by a new bucket formed with the two intermediate voltages. Finally, bunch No. 2 was split out and continuously reflected inside the drifting bucket like a billiard ball, and this beam motion was visible after the 50th turn in Figs. 3(b), 7, and 8. The beam motion resulted from the beam entanglement of the mismatched momentum particles of bunch No. 2 in the drifting bucket and caused emittance blowup and beam loss. The beam spill from the bucket for bunch No. 2 at $\left(t_{\text {in }}, n\right)=$ $(6 \mu \mathrm{s}, 160)$ in Fig. 7 was consistent with the experimental results in Fig. 3(b) and trapped in the other bucket $(5-12 \mu \mathrm{s})$ until the 350th turn [Figs. 7 and 8]. After the 250th turn, however, the main bunch gradually spread out in the bucket $(0.5-4 \mu \mathrm{s})$ (Fig. 7) different from that of the measured beam shown in Fig. 3(b). One possible reason for this beam spread was the accuracy of the induction voltage profiles, which were calculated from the currents through the matching resistances (see Fig. 1 of Ref. [12]) measured with current transformers. This measurement system was not designed to detect such small voltage fluctuations $<10 \mathrm{~V}$-caused by the impedance mismatch-in the inner time interval of $2.5-4 \mu \mathrm{s}$ with high precision. The voltage fluctuations still affected the barrier-bucket shape in the macroparticle simulation. A similar discrepancy was observed in the offDBB as well. Hence, further investigations are necessary to address these discrepancies. Nonetheless, the simulations reasonably reproduced the measured results, especially in the first 250 turns.

As a result of the onDBB, the final beam distribution unfavorably had a filamentation structure with large empty volumes. The final beam survival ratio, excluding the residual gas effects, was 0.75 , which is $23 \%$ lower than that of the SBB. The longitudinal oscillation of the bunch No. 2 core mainly contributed to this beam loss.

\section{Off-momentum drifting bucket scheme}

The evolution of the beam intensity profile and several snapshots of the beam phase-space distribution in the offDBB are shown in Figs. 9 and 10, respectively. The only difference from the onDBB was the appearance of the $2-\mu$ s auxiliary pulses. These long pulses significantly reduced the longitudinal oscillations of bunch No. 2 (Fig. 9), which was observed in the onDBB [Figs. 3(b) 

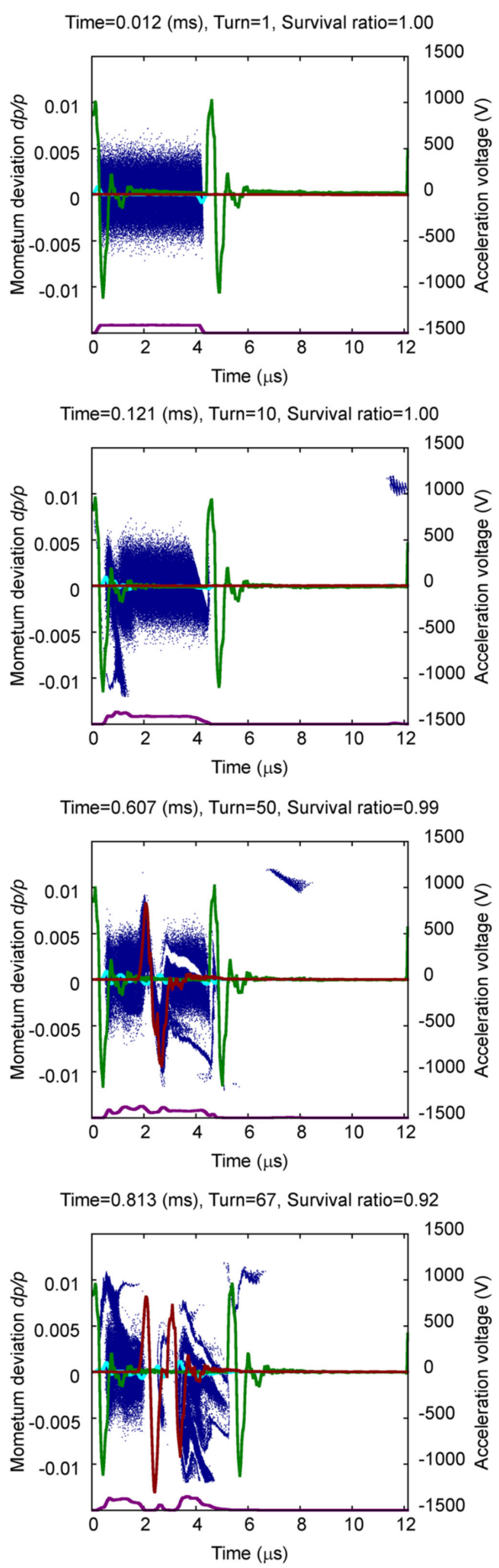

Time=1.214 (ms), Turn=100, Survival ratio=0.88

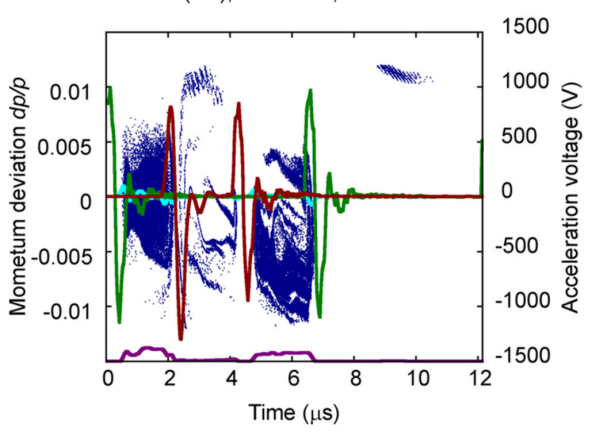

Time $=1.821(\mathrm{~ms})$, Turn $=150$, Survival ratio $=0.86$

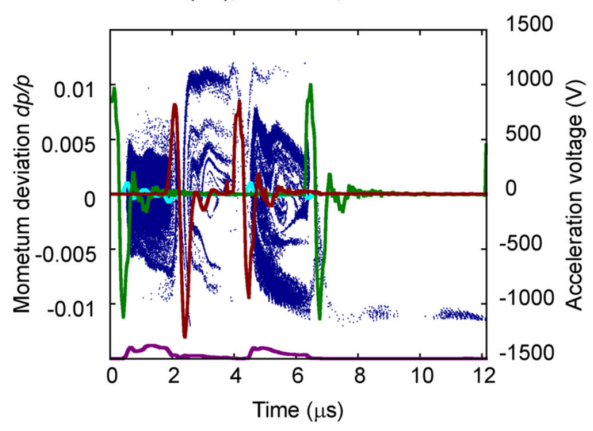

Time=2.403 (ms), Turn=198, Survival ratio=0.77

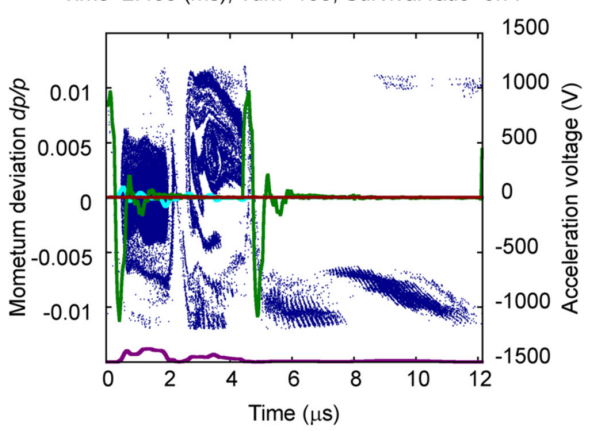

Time $=4.248(\mathrm{~ms})$, Turn=350, Survival ratio $=0.75$

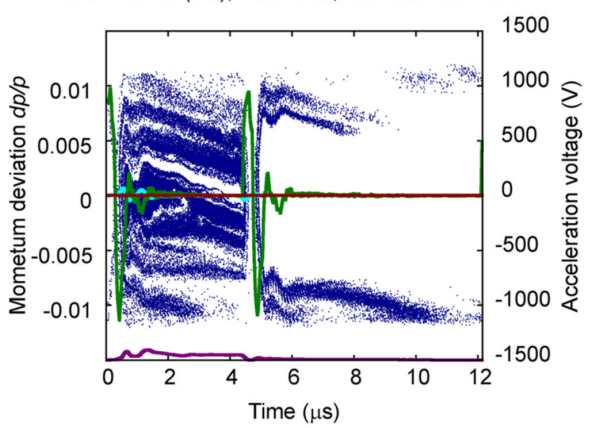

FIG. 8. Snapshots of the beam distribution in the longitudinal phase space in the onDBB at the 1st, 10th, 50th, 67th, 100th, 150th, 198th, and 350th turns, from the top left to the bottom right. The red-colored line indicates the superimposed waveform of the two intermediate voltages. Other colored lines are the same as those in Fig. 5.

and 7], and these results were consistent with the offDBB experimental results [Fig. 3(c)]. There are two main improvements in beam behavior with the offDBB. First, the beam spill of the onDBB at the time $\left(t_{\text {in }}, n\right)=(6 \mu$ s, 160) in Fig. 7 disappeared due to the matched off-momentum beam drifting of bunch No. 2. As a result, the final beam spill located between 5-12 $\mu$ s decreased, as shown in the comparison of Figs. 8 (onDBB) and 10 (offDBB). Second, the final blank space region of the merged bunch was smaller than that of the onDBB. The offDBB, however, caused some unfavorable beam phenomena. In the 197th turn snapshot in Fig. 10, the spilled beam out of the $4-\mu$ s bucket resulted from the abrupt 


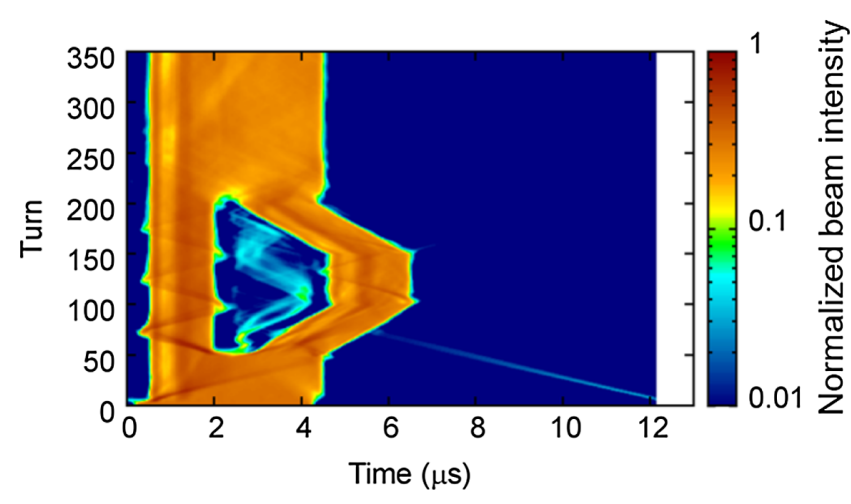

FIG. 9. Evolution of the beam intensity profile in the offDBB (simulation).

appearance of the intermediate voltages around the 50th turn. The sudden absence of the intermediate voltages around the 200th turn generated an additional blank in the bunch structure.

Overall, in the offDBB, there was more effective bunch drifting with the off-momentum bucket handling for bunch No. 2. Note that the drifting beam bucket was not located on the momentum deviation $\Delta p / p=0$ line. During the splitting (coalescing) process during the 48th-97th (148th197th) turns, the beam bucket should have shifted downward (upward) in the momentum direction. The final beam survival ratio, excluding the effects of residual gas interactions, was 0.83 , which is $14 \%$ lower than that of the SBB and $10 \%$ higher than that of the onDBB. This improvement was attributed to the smaller longitudinal oscillation of bunch No. 2 in the offDBB.

\section{Beam survival}

The evolution of the beam survival ratios in the experiments and simulations is shown as solid lines with 95\% confidence regions in translucent colors and as dashed lines, respectively, in Fig. 11. The 95\% confidence regions are calculated from the deviations of the nine-point simple moving average lines of the measured data. The measured data were preprocessed with a $30-\mathrm{kHz}$ high-pass filter, although the filter also introduced slightly nonlinear differential responses. After the 100th turn, without turn-by-turn ground-voltage compensation, the beam survival ratios from the integral of the beam signals have a maximum fluctuation of $\pm 4 \%$; hence, the beam survival ratios with compensation have the same precision. Broken and longdashed-short-dashed lines in Figs. 11(a) and 11(b) show the beam survival ratios with and without the residual gas effects that originated from the electron capture, respectively. The beam lifetime of $12 \mathrm{msec}$ is predicted from the existing data (see Appendix B). The simulation results with this beam loss fairly reproduce the experimental results. We can say that the evolution of the beam survival ratios in the numerical simulations agrees well with the measured data within an error of $8 \%$.
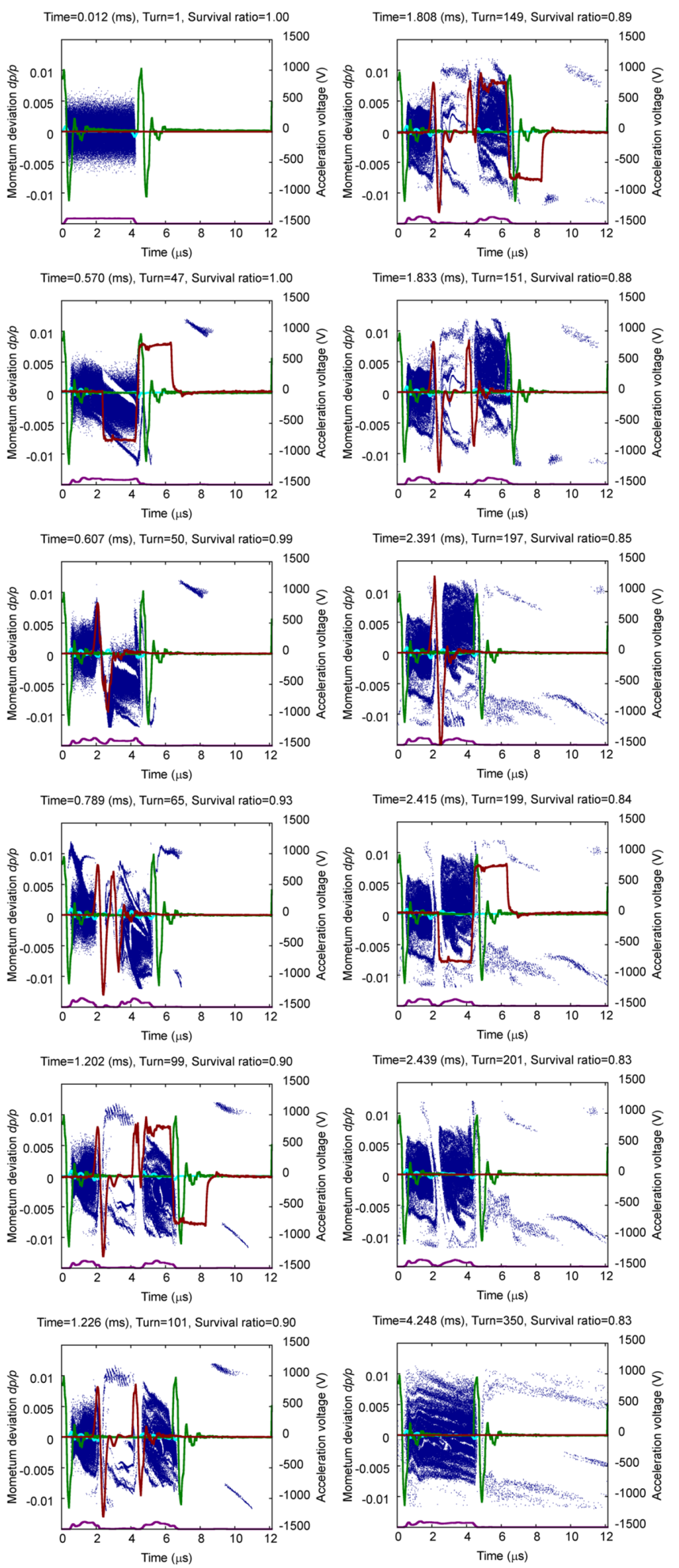

FIG. 10. Snapshots of the beam distribution in the longitudinal phase space in the offDBB at the 1st, 47th, 50th, 65th, 99th, 101st, 149th, 151st, 197th, 199th, 201st, and 350th turns, from the top left to the bottom right. The red-colored line indicates the superimposed waveform of the two intermediate voltages and an auxiliary voltage. Other colored lines are the same as those in Fig. 5. 

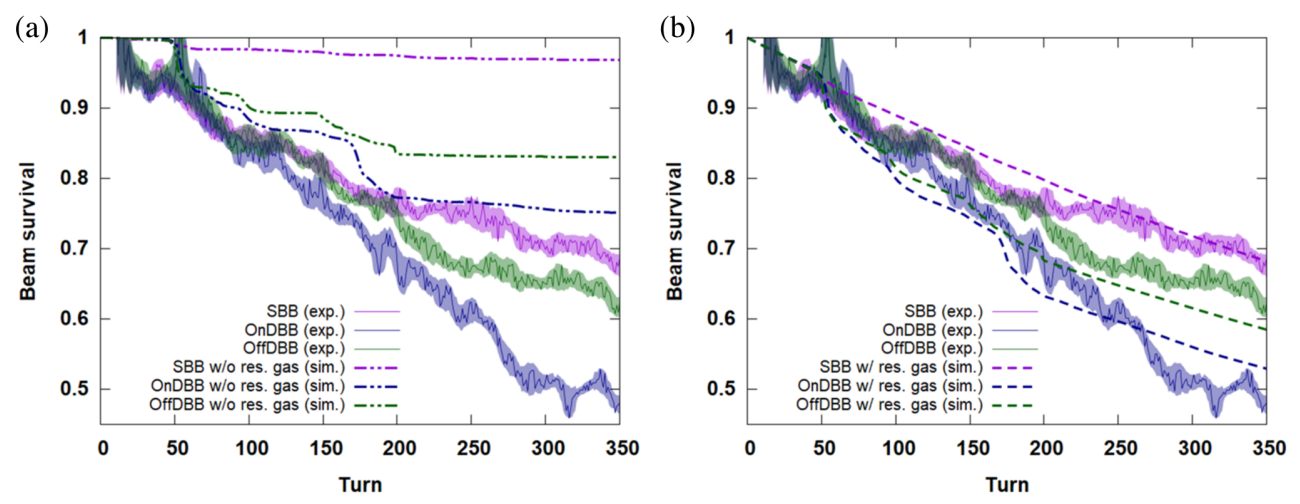

FIG. 11. Beam survival comparison of the experimental and simulation results: (a) without and (b) with beam loss by residual gas interactions.

Beam survival, in general, depends on the transverse beam motion. The present simulation does not take into account any transverse motion, except for the momentum aperture of $\Delta p / p= \pm 0.012$. Nonlinear resonance crossing through the chromaticity is among the possible reasons for beam loss. The above-mentioned error of $8 \%$ may be attributed to these unknown transverse motions.

\section{E. Beam emittance evolution}

We also evaluated the longitudinal emittance evolution in the simulations and performed several comparative analyses to identify the sources of emittance blowup. Figure 12 shows the evolution of longitudinal root-mean-square emittance growths for the SBB, onDBB, and offDBB with and without the space charge effect. The emittance at the $n$th turn, $\varepsilon_{n}$, is calculated based on the beam dynamics information of the position in time and momentum deviation of the particles that are placed in the range between the midpoints of two outer barrier voltage pulses

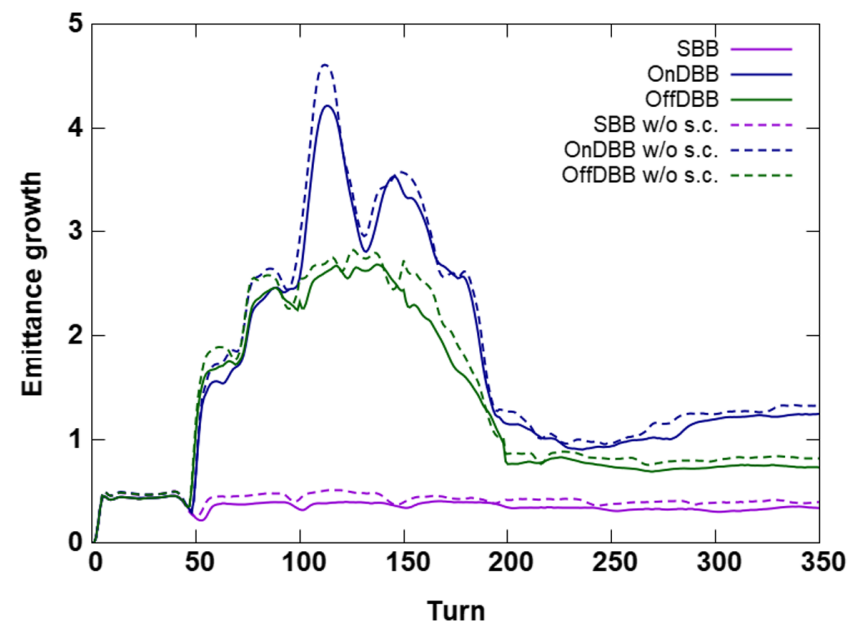

FIG. 12. Evolution of the longitudinal emittance growths in the $\mathrm{SBB}$, onDBB, and offDBB with (solid lines) and without (dashed lines) the space charge effect. (shown in green in Figs. 5, 8, and 10); for instance, the range is $0.4-4.9 \mu$ s every turn in the SBB and 0.4-6.9 $\mu$ s at the 150th turn in the onDBB and offDBB. The emittance growth is defined as $\left(\varepsilon_{n}-\varepsilon_{0}\right) / \varepsilon_{0}$ with the initial beam emittance $\varepsilon_{0}$ at injection.

The following features can be observed in Fig. 12: (1) small emittance blowup at the early stage of barrierbucket handling; (2) abnormally large growth during barrier-bucket handling in the 50th-200th turns for both cases of the onDBB and offDBB; (3) fluctuation of the emittance in the onDBB; (4) apparent discrepancy of the remaining emittance blowup after handling for both cases, as observed at the 350th turn; and (5) negligibly small effects of space charge forces.

All the emittance growths until the 10th turn were $42 \%$ due to the injection mismatch. Further significant emittance growth in the SBB was not observed after the tenth turn. The abnormal emittance growth during the 50th-200th turns can be attributed to the present definition of the emittance, where the vacant space between the two barrier buckets is included. Here, the fluctuation in the emittance for the onDBB is notable. This results from the back-andforth motion of the right bunch core in the bucket, being associated with a loss of particles from the barrier bucket (see Fig. 7), which is caused by nonadiabatic bucket handling beyond Ng's criterion. However, this motion is suppressed in the offDBB (see Fig. 9). This difference is a crucial point demonstrating the advantageous feature of the offDBB that is emphasized in the present paper. The final emittance growths in the SBB, onDBB, and offDBB with (without) the space charge effect were $0.34(0.40)$, 1.25 (1.32), and 0.73 (0.82), respectively. In the practical sense, the emittance after barrier-bucket handling is meaningful and important.

The final emittance in the offDBB was $29 \%$ higher than that in the SBB. This difference should be attributed to the active handling of the barrier bucket as follows: (1) abrupt creation of a pair of barrier voltage pulses around the 50th turn to separate the injected bunch into two parts; (2) absence of the voltage pulses around the 200th turn 
to merge the two separated parts into a single bunch (Figs. 8 and 10).

The creation and absence of barrier voltage pulses is basically nonadiabatic beam handling. Some emittance blowup is inevitable. In addition to the above nonadiabatic beam handling, there are other effects such as (1) bucket distortion due to the reflection voltage induced by the impedance mismatch between the switching power supply and the induction cell [17] and (2) injection mismatch, which are both described in Appendix C. However, because they are not major sources that explain the difference between the onDBB and offDBB, we do not discuss them further.

\section{SUMMARY}

We have demonstrated longitudinal bunch splitting and coalescing with an offDBB in the induction synchrotron. This manipulation was performed beyond the criterion for minimizing longitudinal emittance blowup that the drift speed of buckets must be much lower than the phase drift speed of the maximum off-momentum particles; the reference particle is on-momentum and never moves in the longitudinal phase space. Off-momentum longitudinal beam splitting and coalescing were achieved with less beam loss than the on-momentum schemes in this experiment. The measured results of the longitudinal beam intensity profile, which were reasonably reproduced in the particle simulations, showed the effectiveness of the offmomentum beam handling for longitudinal bunch splitting and coalescing. The emittance blowup realized in the simulations also confirmed the notable advantage of the offDBB over the onDBB, although some emittance blowup was inevitable mainly in the nonadiabatic process of abrupt creation and absence of the inner barrier voltage pulses to

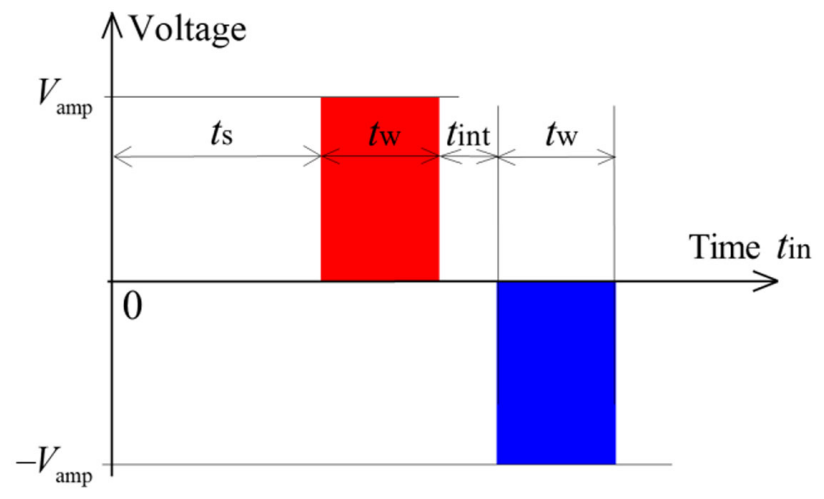

FIG. 13. A typical acceleration pulse of positive and negative voltages.

independently handle two bunches. Although the parameters of the voltage pulse timing or height for creation and absence of the barrier voltage pulses must be optimized by improving the FPGA programming hereafter, the offmomentum scheme that has been discussed in this paper provides a wide variety of beam handling in the longitudinal direction. Fast extraction of a beam fraction well shaped from a long bunch is one of them, in which a rising time of kicker magnets is secured without much perturbation to the residual part of the bunch. Several split beams could be sent to the same beam line or multiple beam lines with different timing in a single acceleration cycle, rendering the industrial use of ion beams cost efficient.

\section{APPENDIX A: ACCELERATION TIMINGS AND AMPLITUDES}

In this Appendix, we describe the acceleration timings and amplitudes of the SBB, onDBB, and offDBB in detail.

TABLE I. Trigger timings and amplitudes of the acceleration pulse in the SBB.

\begin{tabular}{lccccc}
\hline \hline Cell id & Turn $n$ & Start time $t_{s}(\mu \mathrm{s})$ & Width $t_{w}(\mathrm{~ns})$ & Interval $t_{\text {int }}(\mathrm{ns})$ & Volume $V_{\text {amp }}(\mathrm{kV})$ \\
\hline Cell No. 1 & $1-350$ & 0.0 & 250 & 150 & 1.0 \\
Cell No. 2 & $1-350$ & 4.65 & 250 & 150 & 1.0 \\
\hline \hline
\end{tabular}

TABLE II. Trigger timings and amplitudes of the acceleration pulse in the onDBB.

\begin{tabular}{lccccc}
\hline \hline Cell id & Turn $n$ & Start time $t_{s}(\mu \mathrm{s})$ & Width $t_{w}(\mathrm{~ns})$ & Interval $t_{\text {int }}(\mathrm{ns})$ & Volume $V_{\text {amp }}(\mathrm{kV})$ \\
\hline Cell No. 1 & $1-350$ & 0.0 & 250 & 150 & 1.0 \\
Cell No. 2 & $1-47$ & 4.65 & 250 & 150 & 1.0 \\
& $48-97$ & $4.65+(n-47) \times 0.04$ & 250 & 150 & 1.0 \\
& $98-147$ & 6.65 & 250 & 150 & 1.0 \\
& $148-197$ & $4.65+(197-n) \times 0.04$ & 250 & 150 & 1.0 \\
Cell No. 3 & $498-350$ & 4.65 & 250 & 150 & 1.0 \\
Cell No. 4 & $48-97$ & $2.0+(n-74) \times 0.04$ & 250 & 150 & 0.81 \\
& $98-147$ & 4.0 & 250 & 150 & 0.81 \\
& $148-197$ & $2.0+(197-n) \times 0.04$ & 250 & 150 & 0.81 \\
\hline \hline
\end{tabular}


TABLE III. Trigger timings and amplitudes of the acceleration pulse in the offDBB.

\begin{tabular}{lccccc}
\hline \hline Cell id & Turn $n$ & Start time $t_{s}(\mu \mathrm{s})$ & Width $t_{w}(\mathrm{~ns})$ & Interval $t_{\text {int }}(\mathrm{ns})$ & Volume $V_{\mathrm{amp}}(\mathrm{kV})$ \\
\hline Cell No. 1 & $1-350$ & 0.0 & 250 & 150 & 1.0 \\
Cell No. 2 & $1-47$ & 4.65 & 250 & 150 & 1.0 \\
& $48-97$ & $4.65+(n-47) \times 0.04$ & 250 & 150 & 1.0 \\
& $98-147$ & 6.65 & 250 & 150 & 1.0 \\
& $148-197$ & $4.65+(197-n) \times 0.04$ & 250 & 150 & 1.0 \\
& $198-350$ & 4.65 & 250 & 150 & 1.0 \\
Cell No. 3 & $48-197$ & 2.0 & 250 & 150 & 0.81 \\
Cell No. 4 & $48-97$ & $2.0+(n-47) \times 0.04$ & 250 & 150 & 0.81 \\
& $98-147$ & 4.0 & 250 & 150 & 0.81 \\
Cell No. 5 & $148-197$ & $2.0+(197-n) \times 0.04$ & 250 & 150 & -0.81 \\
& $46-47$ & 2.4 & 2000 & 150 & 0.81 \\
& $98-99$ & 4.4 & 2000 & 150 & 0.81 \\
& $148-149$ & 4.4 & 2000 & 150 & -0.81 \\
\hline \hline
\end{tabular}

A set of induction acceleration pulses with voltage amplitude $V_{\text {amp }}$, start time $t_{s}$, pulse width $t_{w}$, and pulse interval $t_{\text {int }}$ is illustrated in Fig. 13. Time $t_{\text {in }}=0$ corresponds to the longitudinal beam phase $\tau=0$ at each turn.

Tables I, II, and III summarize the acceleration timings and amplitudes at each turn in the SBB, onDBB, and offDBB, respectively.

\section{APPENDIX B: RESIDUAL GAS EFFECT}

The numerical beam studies in Sec. IV show that beam loss occurred due to the vacuum level, injection mismatch, and momentum diffusion. Beam loss due to residual gas interaction is unavoidable because of the current achievable vacuum level $P\left(\sim 2.0 \times 10^{-8}\right.$ Torr $)$ of the KEK-DA ring. The evolution of beam survival quantity $N(n)$ [14] is described as

$$
\begin{aligned}
N(n) & =N(0) \cdot \exp \left[-2.12 \times 10^{27} \cdot P(\text { Torr }) \cdot \sigma_{\text {total }} \cdot \beta \cdot T_{0} \cdot n\right] \\
& =N(0) \cdot \exp \left(-\frac{n}{n_{\text {life }}}\right)
\end{aligned}
$$

with

$$
n_{\text {life }}=\frac{1}{2.12 \times 10^{27} \cdot P(\text { torr }) \cdot \beta T_{0} \sigma_{\text {total }}},
$$

where $\sigma_{\text {total }}$ is the sum of the cross sections for electron capture, $\sigma_{\text {cap }}$, and for electron stripping, $\sigma_{\text {strip }}$. We do not have data available for precise residual gas information to identify residual gas species. Therefore, we used $\sigma_{\text {total }}=1.75 \times 10^{-16} \mathrm{~cm}^{2}$, instead, from the data for the residual gas of air, as noted in Fig. 3 of Ref. [18]. With $T_{0}=12.1 \mu \mathrm{s}$ and $\beta=1.03 \times 10^{-2}, n_{\text {life }}$ was estimated to be $\sim 1.1 \times 10^{3}$ turns. The time constant $n_{\text {life }} T_{0} \sim 13 \mathrm{~ms}$ is close to the fit value $(12 \mathrm{~ms})$ in the simulation results in Sec. IV. The next upgrade of the KEK-DA includes the introduction of several ceramic ducts to improve vacuum conditions.

\section{APPENDIX C: OTHER EMITTANCE CALCULATIONS}

Figure 14 shows the effect of the bucket distortion from the impedance mismatch and the technical limitations of the intermediate voltage amplitude $V_{\mathrm{amp}}=0.81 \mathrm{kV}$ for Cell No. 4 (see Tables II and III of Appendix A). For the simulations without distortions, we set the ideal voltage amplitude ( $V_{\mathrm{amp}}=1 \mathrm{kV}$ for cell No. 4) and removed the small fluctuations in the buckets. These effects produced some differences in the 120th-260th turns in the onDBB, although each final emittance difference from the nominal one was $<5 \%$. This result suggests that bucket distortion

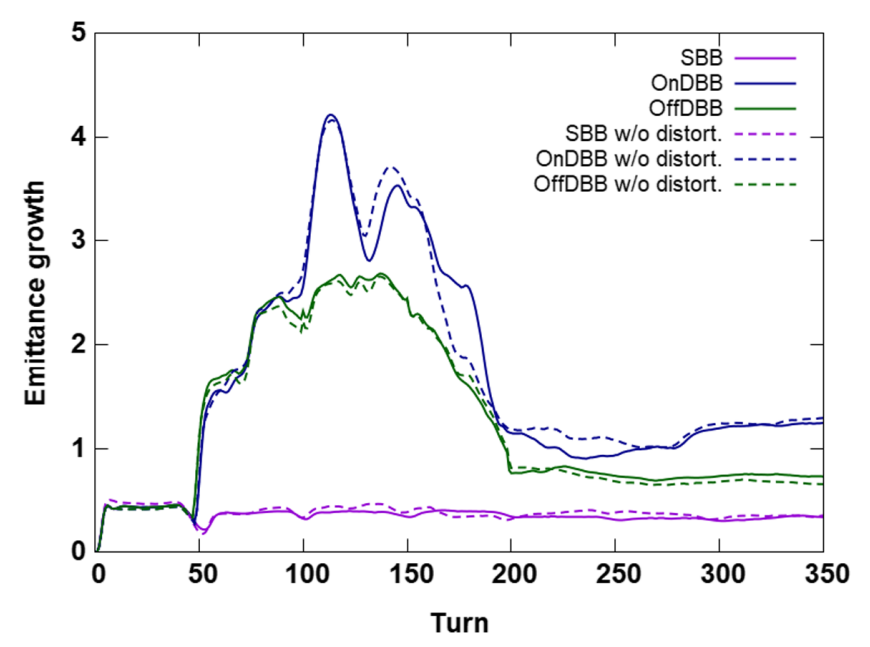

FIG. 14. Evolution of the longitudinal emittance growths in the $\mathrm{SBB}$, onDBB, and offDBB with (solid lines) and without (dashed lines) bucket fluctuations caused by impedance mismatch and the technical limitations. 


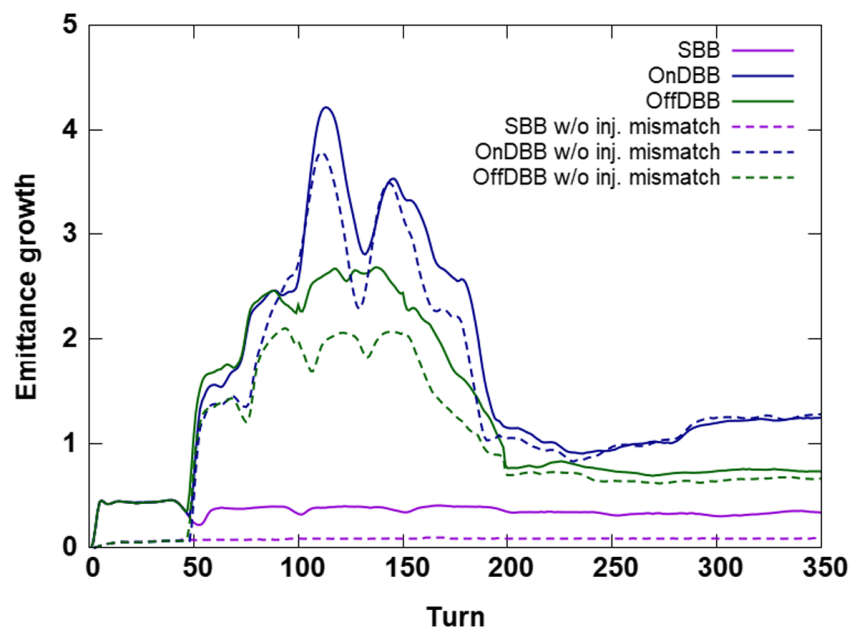

FIG. 15. Evolution of the longitudinal emittance growths in the $\mathrm{SBB}$, onDBB, and offDBB with (solid lines) and without (dashed lines) injection mismatch.

TABLE IV. Final emittance growths in the SBB, onDBB, and offDBB.

\begin{tabular}{lccc}
\hline \hline & SBB & onDBB & offDBB \\
\hline Nominal case & 0.34 & 1.25 & 0.73 \\
Zero space-charge effect & 0.40 & 1.32 & 0.82 \\
Zero distortion barrier bucket & 0.35 & 1.29 & 0.66 \\
Matched injection & 0.09 & 1.28 & 0.66 \\
\hline \hline
\end{tabular}

was not the primary source of overall emittance growth in this study.

The effects of the injection mismatch were analyzed. The injection mismatch caused $\sim 40 \%$ emittance growth in the 0th-10th turns. Hence, we delayed the beam injection by $350 \mathrm{~ns}$ and the right barrier voltage generation by $150 \mathrm{~ns}$ for the simulations without the mismatch. The emittance growth in the 0th-10th turns dropped significantly from 0.42 to 0.04 [Fig. 15], although the final onDBB and offDBB emittance growths were not drastically changed and were 1.28 and 0.66 , respectively, despite the optimized beam injection. Finally, Table IV summarizes the above results of the final emittance growths in the SBB, onDBB, and offDBB.

[1] J. E. Griffin, C. Ankenbrandt, J. A. MacLachlan, and A. Moretti, Isolated bucket rf systems in the Fermilab antiproton facility, IEEE Trans. Nucl. Sci. 30, 3502 (1983).

[2] C. M. Bhat, Applications of bucket systems at Fermilab, in Proceedings of the international workshop on recent progress in induction accelerators (RPIA2006), 2006,
KEK, Japan, KEK Proceedings 2006-12, Report No. FERMILAB-CONF-06-102 AD, pp. 45-59.

[3] M. Fujieda, Y. Iwashita, A. Noda, Y. Mori, C. Ohmori, Y. Sato, M. Blaskiewicz, J. M. Brennan, T. Roser, K. S. Smith, R. Spitz, and A. Zaltsmann, Bucket experiment at the AGS, Phys. Rev. ST Accel. Beams 2, 122001 (1999).

[4] M. Blaskiewicz, J. M. Brennan, T. Roser, K. Smith, R. Spitz, A. Zaltsman, M. Fujieda, Y. Iwashita, A. Noda, M. Yoshii, Y. Mori, C. Ohmori, and Y. Sato, Barrier cavities in the Brookhaven AGS, in Proceedings of the 18th Particle Accelerator Conference, New York, 1999 (IEEE, New York, 1999).

[5] K. Takayama and J. Kishiro, Induction synchrotron, Nucl. Instrum. Methods Phys. Res., Sect. A 451, 304 (2000).

[6] K. Takayama, J. Kishiro, M. Sakuda, Y. Simosaki, and M. Wake, Superbunch Hadron Colliders, Phys. Rev. Lett. 88, 144801 (2002).

[7] Y. Shimosaki, E. Nakamura, K. Takayama, K. Torikai, M. Watanabe, M. Nakajima, and K. Horioka, Beam-dynamic effects of a droop in an induction accelerating voltage, Phys. Rev. ST Accel. Beams 7, 014201 (2004).

[8] K. Takayama et al., Observation of the Acceleration of a Single Bunch by Using the Induction Device in the KEK Proton Synchrotron, Phys. Rev. Lett. 94, 144801 (2005).

[9] K. Takayama, Y. Arakida, T. Dixit, T. Iwashita, T. Kono, E. Nakamura, K. Otsuka, Y. Shimosaki, K. Torikai, and M. Wake, Experimental Demonstration of the Induction Synchrotron, Phys. Rev. Lett. 98, 054801 (2007).

[10] T. Iwashita, T. Adachi, K. Takayama, K. W. Leo, T. Arai, Y. Arakida, M. Hashimoto, E. Kadokura, M. Kawai, T. Kawakubo, T. Kubo, K. Koyama, H. Nakanishi, K. Okazaki, K. Okamura, H. Someya, A. Takagi, A. Tokuchi, and M. Wake, KEK digital accelerator, Phys. Rev. ST Accel. Beams 14, 071301 (2011).

[11] K. Takayama, T. Yoshimoto, M. Barata, L. K. Wah, L. Xingguang, T. Iwashita, S. Harada, T. Adachi, T. Arai, D. Arakawa, H. Asao, E. Kadokura, T. Kawakubo, H. Nakanishi, Y. Okada, K. Okamura, K. Okazaki, A. Takagi, S. Takano, and M. Wake, Induction acceleration of heavy ions in the KEK digital accelerator: Demonstration of a fast-cycling induction synchrotron, Phys. Rev. ST Accel. Beams 17, 010101 (2014).

[12] T. Yoshimoto, M. Hirose, X. Liu, T. Adachi, E. Kadokura, T. Kawakubo, S. Takano, and K. Takayama, Wideband induction acceleration and its intrinsic nature in the KEK digital accelerator, Nucl. Instrum. Methods Phys. Res., Sect. A 797, 191 (2015).

[13] T. Yoshimoto, X. Liu, T. Adachi, T. Kawakubo, H. Kobayashi, S. Takano, E. Kadokura, K. Okamura, and K. Takayama, Super-bunch induction acceleration scheme in the KEK digital accelerator, in Proceedings of the 13th International Conference on Heavy Ion Accelerator Technology (HIAT2015), Yokohama, Japan 2015 (JACoW, Yokohama, 2015).

[14] N. Munemoto, S. Takano, E. Kadokura, Taufik, T. Yoshimoto, X. Liu, T. Adachi, M. Ikeda, T. Kawakubo, K. Okamura, K. Takayama, and M. Wake, Direct injection of fully stripped carbon ions into a fast-cycling induction synchrotron and their capture by the bucket, Phys. Rev. Accel. Beams 20, 080101 (2017). 
[15] K. Y. Ng, Continuous multiple injections at the Fermilab Main Injector, Phys. Rev. ST Accel. Beams 5, 061002 (2002).

[16] K. W. Leo, T. Adachi, T. Arai, and K. Takayama, Einzel lens chopper and behavior of the chopped beam in the KEK digital accelerator, Phys. Rev. ST Accel. Beams 16, 043502 (2013).
[17] K. Koseki and Y. Shimosaki, Parasitic resonance in a solidstate pulse power modulator, Nucl. Instrum. Methods Phys. Res., Sect. A 556, 24 (2006).

[18] S. K. Allison, J. Cuevas, and P. G. Murphy, Experimental cross sections for charge-changing collisions of $\mathrm{He}+$ and $\mathrm{He}++$ ions traversing gases, Phys. Rev. 102, 1041 (1956). 